\title{
Impact of immunohistochemically identified lymphatic invasion on nodal metastasis in early gastric cancer
}

\author{
Akiniro Sako, Joji Kitayama, Makoto Ishikawa, Hiroharu Yamashita, and Hirokazu Nagawa \\ Department of Surgical Oncology, University of Tokyo, 7-3-1 Hongo, Bunkyo-ku, Tokyo 113-8655, Japan
}

\begin{abstract}
Background. Among various clinical and pathological findings, lymphatic invasion ( $\mathrm{Ly}$ ) is the strongest risk factor for nodal metastasis in gastric cancer. However, the diagnosis of Ly is subjective and often inaccurate because of the difficulty of detecting lymphatic vessels with conventional hematoxylin and eosin (HE) staining.

Methods. The distribution of lymphatics in the normal gastric wall was immunohistochemically characterized using a new selective marker of lymphatic endothelium, D2-40, in surgical specimens resected for early gastric cancer (EGC). Then, Ly in the primary lesion was reevaluated, and the positive (PPV) and negative (NPV) predictive values for nodal metastasis were comparatively examined for Ly detected by HE staining (Ly-HE) and by immunohistochemical staining (Ly-IM) in 131 cases of EGC.

Results. D2-40-positive lymphatic vessels were observed in the deep proper mucosal layer, and the lymphatic vessel density (LVD) was extremely high in the muscularis mucosa (MM) layer. The number of Ly-IM-positive cases (15/131) was higher than the Ly-HE-positive cases (10/131). In 48 cases of intestinal-type cancer, Ly-IM had a PPV of 33.3\% (2/6) and anNPV of $100 \%(42 / 42)$, which was more accurate than the corresponding figures for $\mathrm{Ly}-\mathrm{HE}$ (25\% and $98 \%$, respectively). In contrast, the accuracy of Ly-IM was similar to that of $\mathrm{Ly}-\mathrm{HE}$ in 83 cases of diffuse-type cancer.

Conclusion. Lymphatic vessels are most densely distributed in the MM layer in the gastric wall. Immunohistochemical identification of lymphatics is useful to increase the accuracy of diagnosing Ly in resected gastric EGCs. Ly-IM is superior to $\mathrm{Ly}-\mathrm{HE}$ as a predictor of nodal metastasis, at least for intestinal-type EGC.
\end{abstract}

Key words Gastric cancer · Lymphatic invasion · D2-40 • Lymph node metastasis - Histological type

Offprint requests to: A. Sako

Received: April 30, 2006 / Accepted: July 30, 2006

\section{Introduction}

The prognosis of patients with early gastric cancer (EGC), defined as being confined to the mucosa or submucosa irrespective of the presence of regional lymph node (LN) metastasis, has improved with radical gastrectomy with complete removal of regional LN $[1,2]$. However, recent technical improvements in endoscopic mucosal resection (EMR), especially the insulated-tipped (IT) needle knife, have enabled endoscopic en bloc resection of large submucosal lesions $[3,4]$. Although nodal metastasis is one of the most important prognostic factors, the incidence has been reported to be approximately $3 \%$ in intramucosal EGC and $20 \%$ in submucosal EGC $[5,6]$. Therefore, theoretically, many EGCs can be treated with EMR (thereby avoiding radical gastrectomy) and conventional lymphadenectomy.

Numerous studies have reported the risk factors for lymph node metastasis in EGC, including tumor size, depth, histological type, ulceration, and lymphatic invasion (Ly), from the clinicopathological data from cases of surgically resected cancers [5,6]. Assessment of these risk factors, except for Ly, can mostly be achieved by endoscopic investigation. In contrast, evaluation of Ly can be performed only after EMR; and positive Ly commonly requires additional extended surgery based on various criteria for local treatment $[5,7,8]$. Indeed, retrospective data have indicated that the rate of lymphatic metastasis is markedly higher in Ly-positive than in Lynegative EGC $[6,9,10]$. However, the diagnosis of Ly is usually performed on the basis of conventional hematoxylin-eosin (HE) staining, and sometimes lacks objectivity possibly because of the inability to distinguish lymphatics from blood vessels accurately.

Recently, several selective markers of lymphatic endothelium have been identified: podoplanin [11], prox-1 [12], LYVE-1 [13], desmoplakin [14], vascular endothelial growth factor receptor-3 (VEGFR-3) [15,16], and 
D2-40 [17,18]. In this study, we used a new monoclonal antibody (mAb) to D2-40 and immunohistochemically characterized lymphatic vessels in each layer of the normal gastric wall in paraffin sections. Thereafter, we reevaluated Ly of the primary lesion and examined the relation between immunohistochemically identified Ly and lymph node metastasis.

\section{Patients and methods}

\section{Patients}

This study included 131 patients with EGC during 19932001 who underwent curative gastrectomy with standard lymph node dissection in the First Department of Surgery, Tokyo University Hospital. Another 37 advanced gastric cancers (AGC) were examined as well. In all cases with informed consent, serial step sections $3 \mu \mathrm{m}$ thick were cut, fixed in 10\% formalin solution, and embedded in paraffin. All of the resected primary tumors and regional lymph nodes were examined histologically using HE staining according to the Japanese Classification of Gastric Carcinoma [19], except for histological type. Tumors were histologically classified into two types: intestinal type (papillary and tubular adenocarcinoma with no component of diffuse type) and diffuse type (poorly differentiated adenocarcinoma and signet-ring cell carcinoma, including mixed type).

\section{Immunohistochemistry}

Serial 3- $\mu$ m-thick sections were deparaffinized in xylene, hydrated through a graded series of ethanol, and then immersed in 3\% hydrogen peroxide in 100\% methanol for $30 \mathrm{~min}$ to inhibit endogeneous peroxidase activity. To activate the antigens, the sections were boiled in $10 \mathrm{mM}$ citrate buffer $\mathrm{pH} 6.0$ for $30 \mathrm{~min}$. After rinsing in phosphate-buffered saline (PBS), the sections were incubated with normal rabbit serum for $10 \mathrm{~min}$ and then incubated overnight at $4{ }^{\circ} \mathrm{C}$ in humid chambers with a mouse mAb D2-40 (Signet, Dedham, MA, USA) with 1:100 dilution that reacts with human lymphatic endothelium [18,20] or CAM5.2 (Becton Dickinson, San Jose, CA, USA) with 1:8 dilution, which reacts with human cytokeratins 8 and 18 [17]. After washing three times with PBS, the sections were incubated with biotinylated anti-mouse immunoglobulin for $20 \mathrm{~min}$. After washing again with PBS, the slides were treated with peroxidase-conjugated streptavidin tetrahydrochloride for $3 \mathrm{~min}$. Light counterstaining with Mayer's hematoxylin was performed.

\section{Quantification of lymphatic vessels}

The number of D2-40-positive lymphatic vessels was assessed in normal gastric mucosa ( $>3 \mathrm{~cm}$ from the margin of the intramucosal cancer). The gastric wall levels were classified as mucosa (M), muscularis mucosa (MM), submucosa (SM), muscularis propria (MP), and subserosa (SS). The number of lymphatic vessels in a $1 \mathrm{~mm}$-wide portion of each layer was counted, and the height of the layer was measured. Five serial spots were assessed in one sample, and six samples were examined. The mean lymphatic vessel density per square millimeter (LVD) was calculated from 30 data points in each layer.

\section{Evaluation of lymphatic invasion}

Lymphatics were detected by D2-40 staining, and tumor cells in the lymphatics were detected by cytokeratin staining using adjacent sections. Lymphatic invasion detected by $\mathrm{HE}$ staining and by immunostaining was expressed as Ly-HE and Ly-IM, respectively. The absence of lymphatic invasion was expressed as negative Ly and the presence of lymphatic invasion as positive Ly.

\section{Statistical analysis}

The association of LN metastasis with age and tumor size was assessed by Student's $t$-test. Other clinicopathological variables were assessed by the chi-squared test, and lymphatic vessel density was analyzed by analysis of variance (ANOVA), with $P<0.01$ considered significant.

\section{Results}

Detection of lymphatic vessels in normal gastric wall

The specificity of D2-40 for lymphatic vessels was first assessed by immunostaining a normal area apart from the cancer tissue. This revealed positive staining of vessels with irregular morphology, an empty lumen, and thin monolayer endothelium. No vessels reactive with D2-40 contained erythrocytes (Fig. 1A). All these findings were compatible with the typical characteristics of lymphatic vessels.

The lymphatics of the mucosal layer were found in the deep levels of the lamina propria layer (within $300 \mu \mathrm{m}$ from the upper margin of the MM layer) (Fig. 1B). The average numbers of lymphatic vessels in each layer (M, MM, SM, MP, SS) per millimeter width were $1.80,4.17,1.40,1.17$, and 0.83 , respectively; and the average heights (micrometers) of each layer were 863, $129,771,942$, and 144 , respectively. The lymphatics ves- 

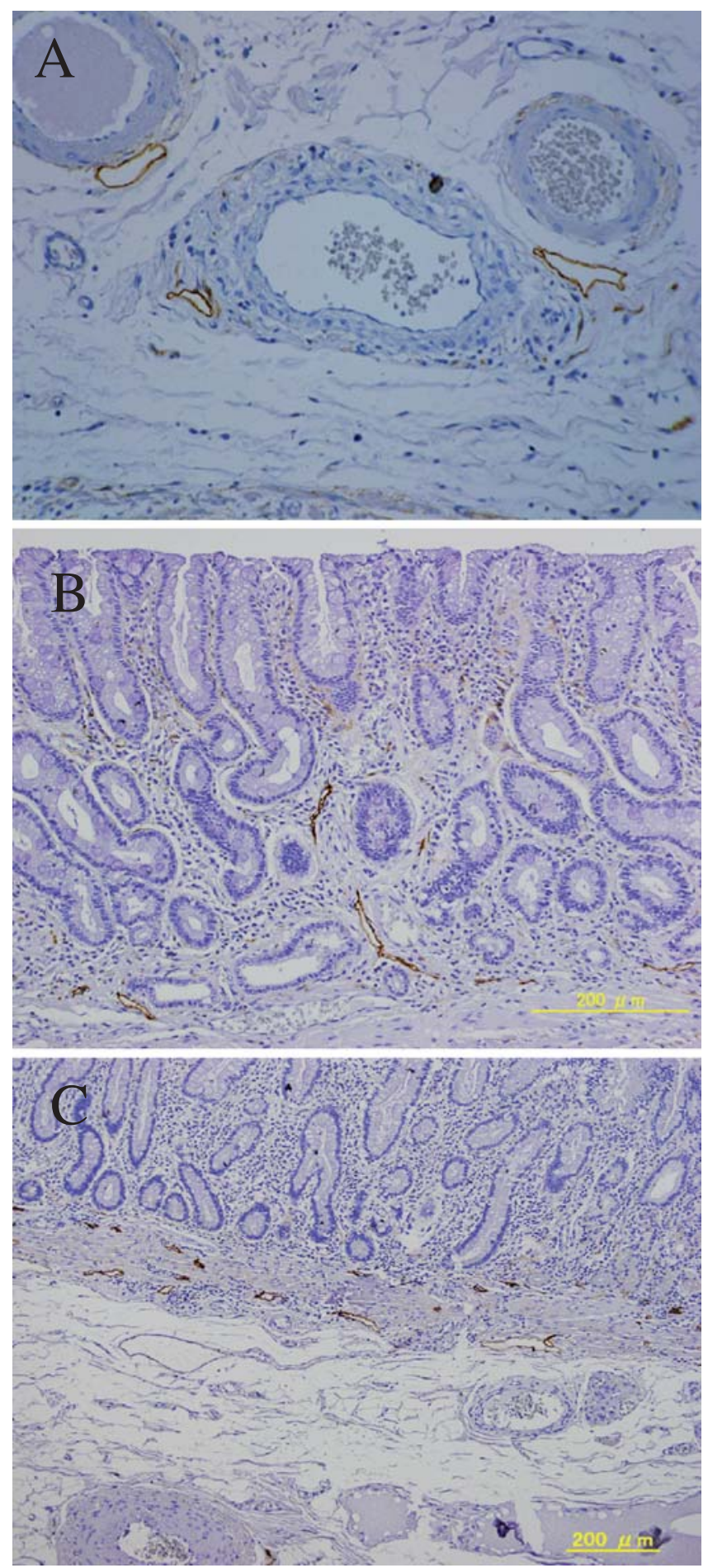

Fig. 1. Staining of normal gastric wall with D2-40. A Negative immunostaining of vascular vessels. $(\times 400)$. B Positive immunostaining of lymphatics in mucosal layer. $(\times 200)$. C Lymphatics in muscularis mucosa and submucosal layer. $(\times 100)$

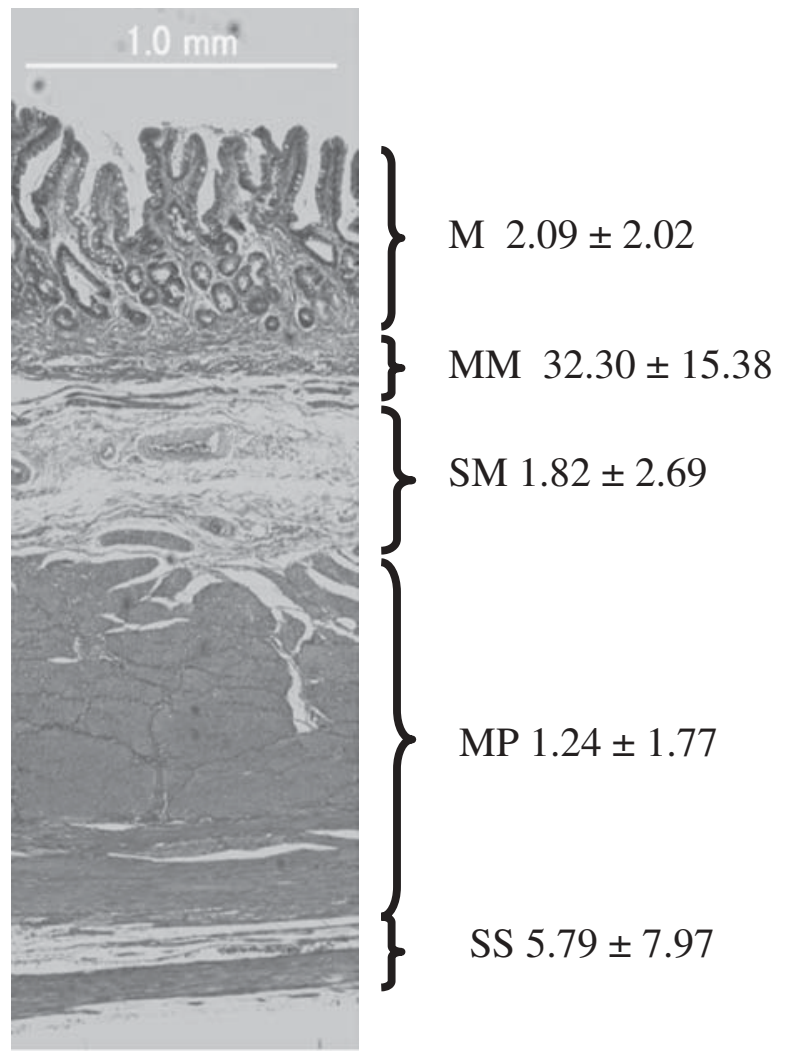

Fig. 2. Lymphatic vessel density (LVD) (per square millimeter) in each layer. Mean \pm SD from 30 spots. LVD is significantly higher in the muscularis mucosa $(M M) . M$, mucosa; $S M$, submucosa; $M P$, muscularis propria; $S S$, subserosa

sel densities (LVDs) were $2.09 \pm 2.02 / \mathrm{mm}^{2}$ (mean \pm SD) and $6.00 \pm 6.08$ in the M layer and the deep levels of the $\mathrm{M}$ layer. In contrast, the MM layer contained a significantly higher number of lymphatics (32.30 \pm $15.38 / \mathrm{mm}^{2}$ ), with no alymphovascular area of more than $0.5 \mathrm{~mm}$, as shown in Fig. 1C. The density of lymphatics found in the SM layer $\left(1.82 \pm 2.69 / \mathrm{mm}^{2}\right)$ was similar to that in the mucosal layer. However, the SM layer also contained alymphovascular areas and lacked lymphatics of more than $5 \mathrm{~mm}$ in length. LVDs in MP and SS were $1.24 \pm 1.77 / \mathrm{mm}^{2}$ and $5.79 \pm 7.97 / \mathrm{mm}^{2}$, respectively (Fig. 2).

\section{Immunohistochemically identified lymphatic invasion}

Lymphatic invasion was clearly demarcated by D2-40 staining, in contrast to HE staining (Fig 3A,B). Ly-IM was assessed by the combination of D2-40-expressing lumen and cytokelatin-expressing tumor cells (Fig. 3C,D). Ly was typically characterized by tumor cells that were completely surrounded by a lymphatic endothelium. In AGC, tumor cells were often observed to show a growing pattern in large lymphatic vessels (Fig. 

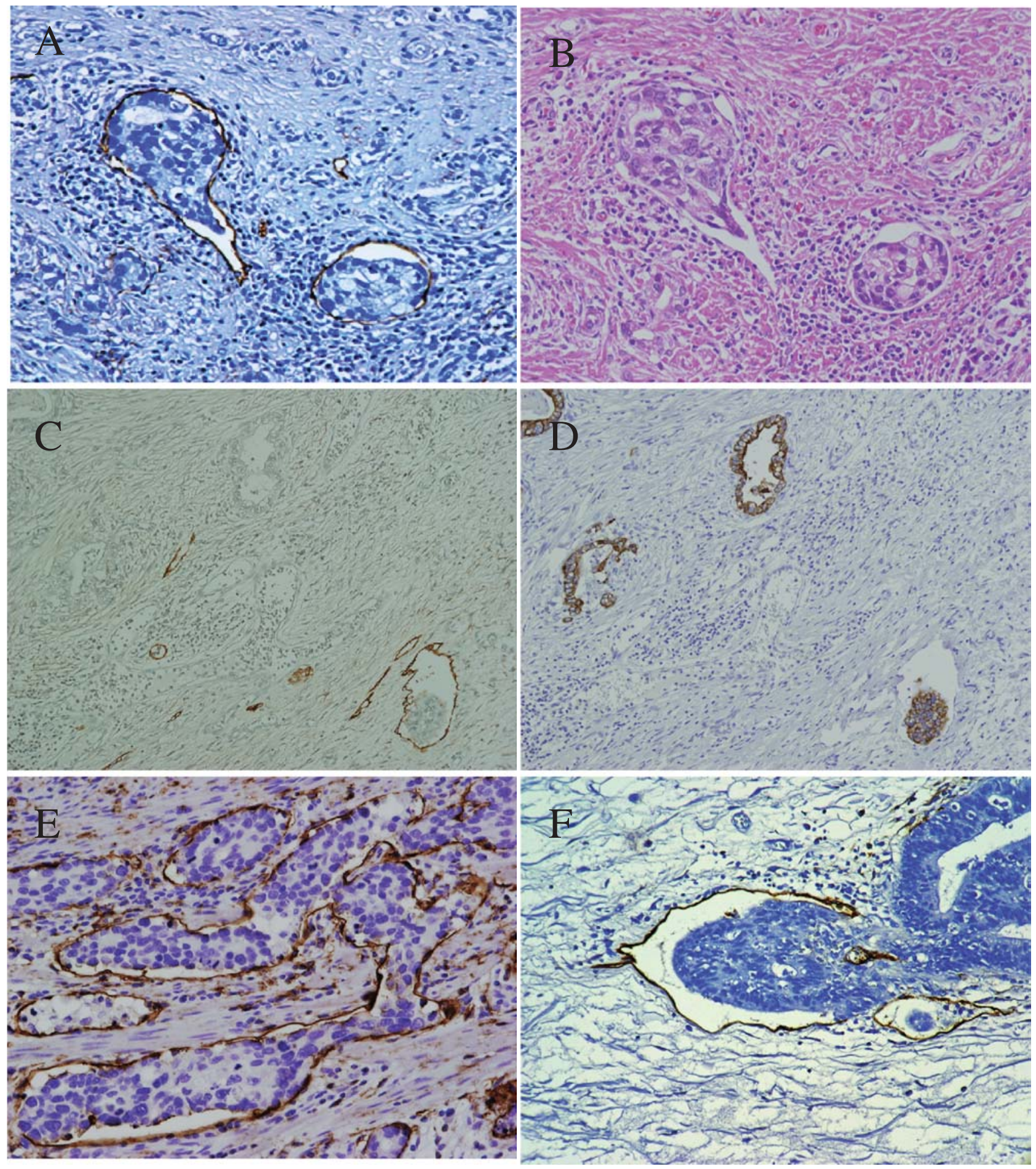

Fig. 3. Identification of lymphatic invasion after immunodetection of lymphatic vessels. A D2-40 staining ( $\times 200)$. B Staining of section adjacent to $\mathbf{A}(\times 200)$. C Positive immunostaining of lymphatics $(\times 100)$. D Positive immunostaining of tumor cells with cytokeratin in the adjacent section $(\times 100)$. E Tumor spreading in lymphatics $(\times 400)$. F Tumor invading the lymphatic wall $(\times 200)$ 
Table 1. Clinicopathological factors and lymph node metastasis of early gastric cancer

\begin{tabular}{lccc}
\hline & \multicolumn{2}{c}{ Lymph node metastasis } \\
\cline { 2 - 4 } Factor & Negative & Positive & $P$ \\
\hline Age & $60.8 \pm 10.5$ & $53.6 \pm 8.5$ & 0.006 \\
Sex & & & \\
$\quad$ Male & $86(87.8 \%)$ & $12(12.2 \%)$ & $\mathrm{NS}$ \\
Female & $27(81.8 \%)$ & $6(18.2 \%)$ & \\
Depth & $63(99.98 \%)$ & $1(0.02 \%)$ & 0.001 \\
$\quad$ m & $51(75.0 \%)$ & $17(25.0 \%)$ & \\
sm & $16(84.2 \%)$ & $3(15.8 \%)$ & $\mathrm{NS}$ \\
Macroscopic & $97(86.6 \%)$ & $15(13.4 \%)$ & 0.005 \\
$\quad$ Elevated & $3.8 \pm 2.8$ & $5.8 \pm 3.0$ & \\
$\quad$ Ulcerated & $46(95.8 \%)$ & $2(4.2 \%)$ & 0.015 \\
Size & $67(80.7 \%)$ & $16(19.3 \%)$ & \\
Histology & $110(91.0 \%)$ & $11(9.0 \%)$ & 0.001 \\
$\quad$ Intestinal & $3(30.0 \%)$ & $7(70.0 \%)$ & \\
Diffuse & $106(86.9 \%)$ & $16(13.1 \%)$ & $\mathrm{NS}$ \\
Lymphatic invasion (HE) & $7(77.8 \%)$ & $2(22.2 \%)$ & \\
$\quad$ Negative & Positive & &
\end{tabular}

Age and size: average \pm standard deviation (SD)

Age and size were evaluated by Student's $t$-test, the others by the chi-squared test

Table 2. Relation between Ly-IM and Ly-HE in early gastric cancer

\begin{tabular}{lcc}
\hline Ly-IM staining & Ly-HE negative & Ly-HE positive \\
\hline Ly-IM negative & 113 & 3 \\
Ly-IM positive & 8 & 7
\end{tabular}

Ly, lymphatic invasion; HE, hematoxylin and eosin staining; IM, immunohistochemical staining

3E). In one case, we could detect tumor cells that were clearly invading the lumen of a lymphatic vessel through its wall (Fig. 3F).

\section{Lymphatic invasion detected by hematoxylin-eosin or immunohistochemical staining}

The clinical and pathological characteristics of 131 patients with EGC are summarized in Table 1. In addition to size, depth, and histology, Ly diagnosed both by HE staining (Ly-HE) and IM staining (Ly-IM) was significantly correlated with LN metastasis. Table 2 shows the positive correlation between Ly-HE and Ly-IM. However, among the 121 EGCs diagnosed as Ly-negative by HE staining, 8 showed positive lymphatic invasion after immunodetection of lymphatics. In some cases, HE diagnosis failed to identify surrounding lymphatics that were filled with tumor emboli (Fig. 4A), and in other cases $\mathrm{HE}$ diagnosis could not distinguish retraction artifacts that isolated tumor aggregates due to tissue shrinkage during fixation from true tumor emboli in lymphovascular spaces (Fig. 4B). In contrast, among the 10 Ly-positive tumors by HE diagnosis, 3 were diagnosed as Ly-negative by IM staining. In these cases, blood vessel invasion was misdiagnosed as lymphatic invasion.

The layers of lymphatic invasion observed in immunostained samples of 15 Ly-IM-positive cases (3 mucosal cancers, 12 submucosal cancers) are expressed in Table 3. In nine of these submucosal cancers, lymphatic invasion by tumor cells was found in the MM layer. Consistent with the distribution of lymphatics, lymphatic invasion was most frequently observed in the MM layer.

\section{Predictive value of Ly-IM for nodal metastasis}

The predictive value of nodal metastasis by Ly-IM in intestinal- and diffuse-type EGCs is shown in Table 4. Among 48 patients with intestinal-type cancer, 6 showed positive Ly-IM, 2 of whom were positive for lymph node metastasis; none of the 42 EGCs with negative Ly-IM showed lymphatic metastasis. Thus, the positive (PPV) and negative (NPV) predictive values were $33 \%(2 / 6)$ and $100 \%$ (42/42), respectively. These values were slightly higher than those of Ly-HE $(25 \%$, $98 \%$ ) In contrast, Ly-IM showed a PPV of $67 \%(6 / 9)$ and an NPV of $87 \%(64 / 74)$ in diffuse-type cancers. This was not superior to the accuracy of Ly-HE in diffusetype EGC. 

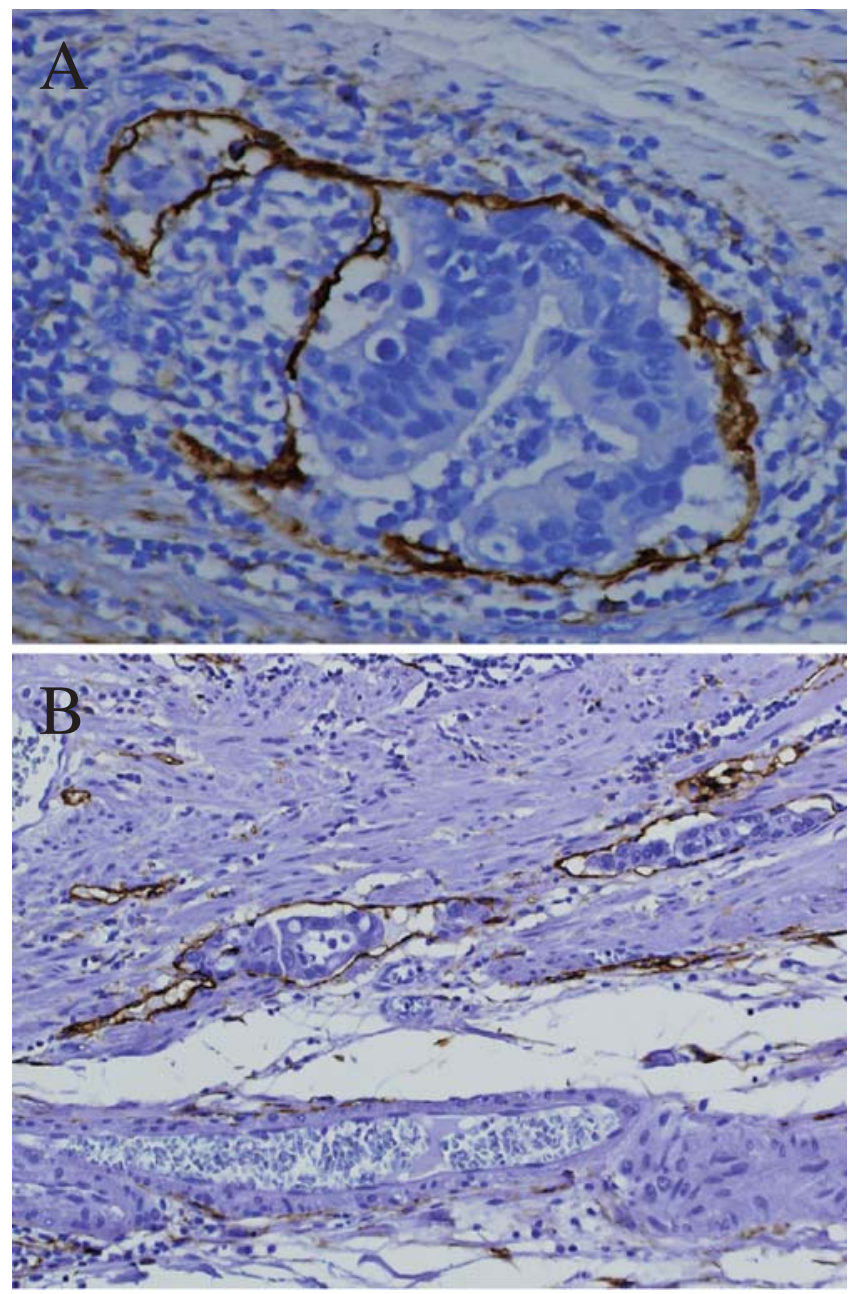

Fig. 4. Typical cases of lymphatic invasion missed with hematoxylin and eosin staining but detected after D2-40 staining. A Tumor emboli in lumen of lymphatics $(\times 200)$. B Lymphatic invasion with retraction artifact $(\times 100)$

\section{Discussion}

D2-40 is an mAb against a 40000-dalton O-linked sialoglycoprotein that was initially identified as the testicular oncofetal antigen [21]. Recent reports, however, have shown that the antibody reacts with a fixation-resistant epitope in lymphatic endothelium in various sites, including malignant tissues [18,22-24]. Our study of the stomach demonstrates that lymphatic microvessels can be clearly observed in the deeper part, but not in the upper area, of the M layer and were most densely distributed in the MM layer in normal gastric mucosa. In the SM layer, the density of lymphatic vessels was mostly the same as that in the M and MP layers. Previous transmission electron microscopy studies demonstrated that lymph capillaries were detected only in the deep lamina propria adjacent to and within the MM layer, although large lymph vessels were observed in the
Table 3. Layer where lymphatic invasion was detected immunohistochemically

\begin{tabular}{ccccc}
\hline & & \multicolumn{3}{c}{ Part of lymphatic } \\
\cline { 3 - 5 } Case & $\begin{array}{c}\text { Depth of } \\
\text { invasion }\end{array}$ & M & MM & SM \\
\hline 1 & m & + & - & \\
2 & m & + & - & \\
3 & m & - & + & \\
4 & sm & + & + & - \\
5 & sm & + & + & - \\
6 & sm & - & + & - \\
7 & sm & - & + & - \\
8 & sm & - & + & - \\
9 & sm & - & + & - \\
10 & sm & - & + & - \\
11 & sm & - & + & - \\
12 & sm & - & + & - \\
13 & sm & + & - & + \\
14 & sm & + & - & + \\
15 & sm & - & - & + \\
\hline
\end{tabular}

+, lymphatic invasion positive; -, lymphatic invasion negative; $\mathrm{m}, \mathrm{M}$, mucosa; sm, SM, submucosa; MM, muscularis mucosa

Table 4. Ly-IM and lymph node metastasis from intestinaltype and diffuse-type early gastric cancer

\begin{tabular}{lccc}
\hline & \multicolumn{2}{c}{ Lymph node metastasis } \\
\cline { 2 - 4 } Cancer type & Negative & Positive & $P^{*}$ \\
\hline Intestinal & & & \\
$\quad$ Ly-IM negative & $42(100 \%)$ & 0 & \\
Ly-IM positive & $4(66.7 \%)$ & $2(33.3 \%)$ & 0.001 \\
Diffuse & & & \\
$\quad$ Ly-IM negative & $64(86.5 \%)$ & $10(13.5 \%)$ & \\
Ly-IM positive & $3(33.3 \%)$ & $6(66.7 \%)$ & 0.001 \\
\hline
\end{tabular}

*By chi-squared test

SM layer [25,26]. Our results are mostly consistent with those data, indicating that the D2-40 antigen is similarly expressed in both lymphatic capillaries and endothelium of larger lymphatics. These findings also suggest that mucosal lymphatic capillaries form the submucosal collecting lymphatics through a close network in the MM layer in the gastric wall.

Lymphatic spread of cancer is assumed to occur through cancer cells permeating into peritumorous lymphatics and reaching the regional lymph nodes. Thus, close contact between tumor cells and lymphatics is thought to be a step in lymphatic metastasis. Clinical studies on gastric cancer have shown that lymphatic metastasis was rare in mucosal cancer but was markedly increased in submucosal cancer [5,6]. This is reasonably explained by our results suggesting that cancer cells invading the MM layer have the most frequent chance to penetrate the lymphatic lumen. In fact, cancer cells invading lymphatic vessels were most frequently detected in the MM layer in our observation (Table 3). 
Recent studies with more detailed pathological analysis have indicated that the depth $[27,28]$ and total volume [29] of submucosal invasion are closely correlated with the risk of lymphatic metastasis. However, our results suggest that the width or area of MM invasion may be more important for the evaluation of nodal status in submucosal gastric cancer.

In our series, we found some inconsistency between Ly-HE and Ly-IM. By immunodetection of lymphatic vessels with D2-40, Ly was additionally detected in 8 of 121 cases that were categorized as Ly-HE-negative, whereas 3 of 10 cases that were categorized as Ly-HEpositive turned out to be from blood vessel invasion. When Ly-IM was evaluated from the standpoint of the predictive value, Ly-IM clearly predicted nodal status more accurately in intestinal-type cancers, with 100\% NPV. We evaluated 11 intestinal-type advanced gastric cancers (AGCs) among 37 AGCs and found that none of the negative Ly-IM AGCs was associated with lymphatic metastasis (data not shown). Therefore, negative Ly-IM is considered to be a reliable marker for no nodal metastasis in intestinal cancer.

In contrast, Ly-IM was not apparently superior to LyHE in diffuse-type cancers. With our method, most Ly was observed as tumor cells that were completely surrounded by lymphatics, whereas tumor cells invading a lymphatic vessel could rarely be found. Therefore, Ly as shown in Fig. 3A or Fig. 3E may be intralymphatic growth characteristic of intestinal-type cancer, as shown in the schema of Fig. 5, because intestinal-type cancers are apt to maintain intercellular contact more frequently than the diffuse type. On the other hand, diffuse-type cancers often lose glandular formation in the submu-

\section{Intestinal type}

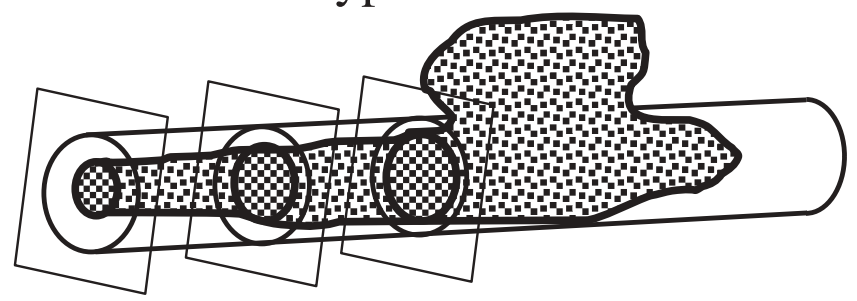

\section{Diffuse type}

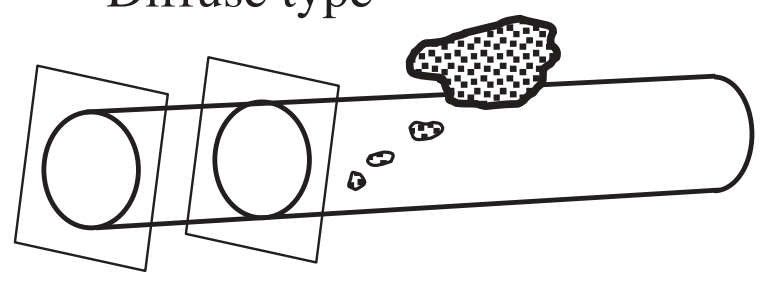

Fig. 5. Schema of lymphatic invasion. Lymphatic invasion of diffuse type has less chance to be detected morphologically cosal layer, and thus tumor cells may be more scattered in lymphatic vessels. Therefore, the morphological detection of intralymphatic tumor cells may be more difficult in diffuse-type than intestinal-type cancers, even though the lymphatic vessels are clearly detected on immunohistochemical staining.

\section{Conclusion}

Lymphatic vessels in the gastric wall can be clearly and selectively identified by immunostaining for D2-40. Because lymphatics are most densely distributed in the MM layer, cancer cells appear to have the greatest chance to penetrate the lymphatics in the MM layer. Pathological evaluation of the area and length of MM invasion might be another important factor to predict nodal metastasis in EGCs. Selective identification of lymphatic vessels with this method can increase the accuracy of detecting lymphatic invasion by the primary tumor, and Ly detected by this method (Ly-IM) is an excellent predictor of nodal metastasis from intestinaltype EGCs. Our data suggest the clinical implication that intestinal-type EGCs, even those with submucosal invasion, might be safely treated with EMR alone when Ly-IM is not observed in the resected material.

Acknowledgments This work was supported by a Grant-in-Aid for Scientific Research from the Ministry of Education, Science, Sports, and Culture of Japan and by a grant from the Ministry of Health and Welfare of Japan.

\section{References}

1. Sano T, Sasako M, Kinoshita T, Maruyama K. Recurrence of early gastric cancer: follow-up of 1475 patients and review of the Japanese literature. Cancer. 1993;72:3174-8.

2. Sue-Ling HM, Martin I, Griffith J, Ward DC, Quirke P, Dixon MF, et al. Early gastric cancer: 46 cases treated in one surgical department. Gut 1992;33:1318-22.

3. Miyamoto S, Muto M, Hamamoto Y, Boku N, Ohtsu A, Baba S, et al. A new technique for endoscopic mucosal resection with an insulated-tip electrosurgical knife improves the completeness of resection of intramucosal gastric neoplasms. Gastrointest Endosc 2002;55:576-81.

4. Ohkuwa M, Hosokawa K, Boku N, Ohtu A, Tajiri H, Yoshida S. New endoscopic treatment for intramucosal gastric tumors using an insulated-tip diathermic knife. Endoscopy 2001;33:221-6.

5. Gotoda T, Yanagisawa A, Sasako M, Ono H, Nakanishi Y, Shimoda $\mathrm{T}$, et al. Incidence of lymph node metastasis from early gastric cancer: estimation with a large number of cases at two large centers. Gastric Cancer 2000;3:219-25.

6. Maehara Y, Orita H, Okuyama T, Moriguchi S, Tsujitani S, Korenaga D, et al. Predictors of lymph node metastasis in early gastric cancer. Br J Surg 1992;79:245-7.

7. Yamao T, Shirao K, Ono H, Kondo H, Saito D, Yamaguchi H, et al. Risk factors for lymph node metastasis from intramucosal gastric carcinoma. Cancer 1996;77:602-6. 
8. Gotoda T, Sasako M, Ono H, Katai H, Sano T, Shimoda T. Evaluation of the necessity for gastrectomy with lymph node dissection for patients with submucosal invasive gastric cancer. $\mathrm{Br}$ J Surg 2001;88:444-9.

9. Song SY, Park S, Kim S, Son HJ, Rhee JC. Characteristics of intramucosal gastric carcinoma with lymph node metastatic disease. Histopathology 2004;44:437-44.

10. Son HJ, Song SY, Kim S, Noh JH, Sohn TS, Kim DS, et al. Characteristics of submucosal gastric carcinoma with lymph node metastatic disease. Histopathology 2005;46:158-65.

11. Breiteneder-Geleff S, Soleiman A, Kowalski H, Horvat R, Amann G, Kriehuber E, et al. Angiosarcomas express mixed endothelial phenotypes of blood and lymphatic capillaries: podoplanin as a specific marker for lymphatic endothelium. Am J Pathol 1999;154:385-94.

12. Wigle JT, Oliver G. Prox1 function is required for the development of the murine lymphatic system. Cell 1999;98:769-78.

13. Banerji S, Ni J, Wang SX, Clasper S, Su J, Tammi R, et al. LYVE1 , a new homologue of the CD44 glycoprotein, is a lymph-specific receptor for hyaluronan. J Cell Biol 1999;144:789-801.

14. Ebata N, Nodasaka Y, Sawa Y, Yamaoka Y, Makino S, Totsuka $\mathrm{Y}$, et al. Desmoplakin as a specific marker of lymphatic vessels. Microvasc Res 2001;61:40-8.

15. Kaipainen A, Korhonen J, Mustonen T, Van Hinsbergh VW, Fang GH, Dumont D, et al. Expression of the fms-like tyrosine kinase 4 gene becomes restricted to lymphatic endothelium during development. Proc Natl Acad Sci U S A 1995;92:3566-70.

16. Joukov V, Pajusola K, Kaipainen A, Chilov D, Lahtinen I, Kukk $\mathrm{E}$, et al. A novel vascular endothelial growth factor, VEGF-C, is a ligand for the Flt4 (VEGFR-3) and KDR (VEGFR-2) receptor tyrosine kinases. EMBO J 1996;15:290-98.

17. Makin CA, Bobrow LG, Bodmer WF. Monoclonal antibody to cytokeratin for use in routine histopathology. J Clin Pathol 1984;37:975-83.

18. Kahn HJ, Marks A. A new monoclonal antibody, D2-40, for detection of lymphatic invasion in primary tumors. Lab Invest 2002;82:1255-7.
19. Japanese Gastric Cancer Association. Japanese Classification of Gastric Carcinoma — 2nd English Edition. Gastric Cancer 1998;1: $10-24$.

20. Kahn HJ, Bailey D, Marks A. Monoclonal antibody D2-40, a new marker of lymphatic endothelium, reacts with Kaposi's sarcoma and a subset of angiosarcomas. Mod Pathol 2002;15:434-40.

21. Marks A, Sutherland DR, Bailey D, Iglesias J, Law J, Lei M, et al. Characterization and distribution of an oncofetal antigen (M2A antigen) expressed on testicular germ cell tumours. Br J Cancer 1999;80:569-78.

22. Franchi A, Gallo O, Massi D, Baroni G, Santucci M. Tumor lymphangiogenesis in head and neck squamous cell carcinoma: a morphometric study with clinical correlations. Cancer 2004;101: 973-8.

23. Niakosari F, Kahn HJ, Marks A, From L. Detection of lymphatic invasion in primary melanoma with monoclonal antibody D2-40: a new selective immunohistochemical marker of lymphatic endothelium. Arch Dermatol 2005;141:440-4.

24. Fogt F, Zimmerman RL, Ross HM, Daly T, Gausas RE. Identification of lymphatic vessels in malignant, adenomatous and normal colonic mucosa using the novel immunostain D2-40. Oncol Rep 2004;11:47-50.

25. Lehnert T, Erlandson RA, Decosse JJ. Lymph and blood capillaries of the human gastric mucosa: a morphologic basis for metastasis in early gastric carcinoma. Gastroenterology 1985;89:939-50.

26. Listrom MB, Fenoglio-Preiser CM. Lymphatic distribution of the stomach in normal, inflammatory, hyperplastic, and neoplastic tissue. Gastroenterology 1987;93:506-14.

27. Cai J, Ikeguchi M, Maeta M, Kaibara N. Micrometastasis in lymph nodes and microinvasion of the muscularis propria in primary lesions of submucosal gastric cancer. Surgery 2000;127:32-9.

28. Kurihara N, Kubota T, Otani Y, Ohgami M, Kumai K, Sugiura H, et al. Lymph node metastasis of early gastric cancer with submucosal invasion. Br J Surg 1998;85:835-9.

29. Matsuzaki H, Kikuchi S, Kakita A. Evaluation of the morphology of submucosal tumor invasion and its volume in early gastric cancer. In Vivo 2003;17:41-4. 